\title{
Research and Application of Mobile Geographic Information Service Technology Based on JSP

\author{
Chengtong $\mathrm{GUO}^{1, \mathrm{a}}$, Yan $\mathrm{YAO}^{1, \mathrm{~b}}$
}

${ }^{1}$ Beijing University of Posts and Telecommunications No.10 Xitucheng Road, Haidian District, Beijing, China

a609323724@qq.com, byaoyan_bupt@sina.com

Keywords: Geographic Information System, JSP, Data Visualization, HTML 5

\begin{abstract}
In this paper, scientific statistical data and geographic location information are combined. A JSP design scheme of mobile geographic information service system is put forward. This system displays statistical data on the map in many ways. Besides, HTML5 technology is also used to achieve a visual, vivid and interactive effect of data presentation. This paper gives a systematic and concrete design scheme and a detailed account of the implementation of dotted information and hierarchical maps presentation, as well as the visualization principle of statistical information. Finally, combined with the actual application effect, it is proved that this design is reasonable, the visualization effect of data is good and the usability is high.
\end{abstract}

\section{Introduction}

The society today is in an era of information. Our work and life are surrounded by all kinds of data. Many statistical data (such as population density, aging, number of school teachers and students, number and funding of R\&D institutions, economic development indicators, etc.) are closely related to geographical position [1]. How to display statistical data with location information intensively and improve the response speed and decision-making ability of relevant departments has become a problem to be solved by various management departments.

By developing a management and service system of scientific statistical data, this topic classifies and analyzes data, combines with Cartogram and displays data visually and solves the existing problems of scattered data, lack of availability and slow processing speed, etc. It can solve the special and general requirements of $R \& D$ institutions for scientific statistical data. Research on mobile geographic information service technology based on JSP provides a technical channel to implement an efficient way that geospatial information services the society, organizes statistical data and displays them in the form of images and graphics in combination with maps, to improve the response speed and decision-making ability of relevant departments. This method is more vivid and visual for users, too.

\section{Overview of the System}

Introduction to JSP technology. JSP (Java Server Pages) is a dynamic web technical standard advocated by Sun Microsystems, established by many companies together. JSP technology is similar to ASP technology. It is to insert Java program segments and JSP tags into traditional web HTML files (*.htm, *.html) and form JSP files (*.jsp) [2]. Web applications developed with JSP is cross-platform, i.e., they can be run under Linux, or other operating systems.

JSP has the following advantages:

1) It can be written once and run anywhere. With respect to this feature, Java has more advantages than PHP. Apart from the system, there is no need to modify codes.

2) Multi-platform support from the system. JSP can be developed in any environment on all platforms. It can be deployed systematically and expanded in any environment. Compared with the limitations of ASP/PHP, it has obvious advantages. 
3) Strong scalability. From running Servlet/JSP with only a small Jar file to multiple servers performing clustering and load balancing and multiple applications processing transactions and information, from one server to countless servers, Java shows great vitality.

4) Diversification and powerful development tool support. Similar to ASP, Java has already owned many excellent development tools, and many of them are free. Besides, they can be run on multiple platforms smoothly.

Mobile geographic information service system should be convenient. It can't be restricted to a specific system or platform, but realizes cross-platform and cross-system use and meet users' demands to access data information in anytime and anywhere on tools like PC, PAD and mobile phones. Based on the above characteristics of JSP, the designed system full meets the demands of such designs. In addition, mature technology is used and service-oriented mobile Internet technology is widely applied in the process of informationization, which also ensures the stability of system.

Design of the system framework. The system based on JSP uses Spring MVC MyBatis framework. MyBatis is an excellent persistence layer framework that supports ordinary SQL queries, stored procedure and advanced mapping. MyBatis eliminates almost all of the manual settings of JDBC codes and parameters, as well as the retrieval encapsulation of result sets [3].MyBatis can use simple XML or annotations for configuration and original mapping and map POJOs (Plain Old Java Objects) of interfaces and Java to the records in the database. The overall software framework of the system is shown in figure 1 :

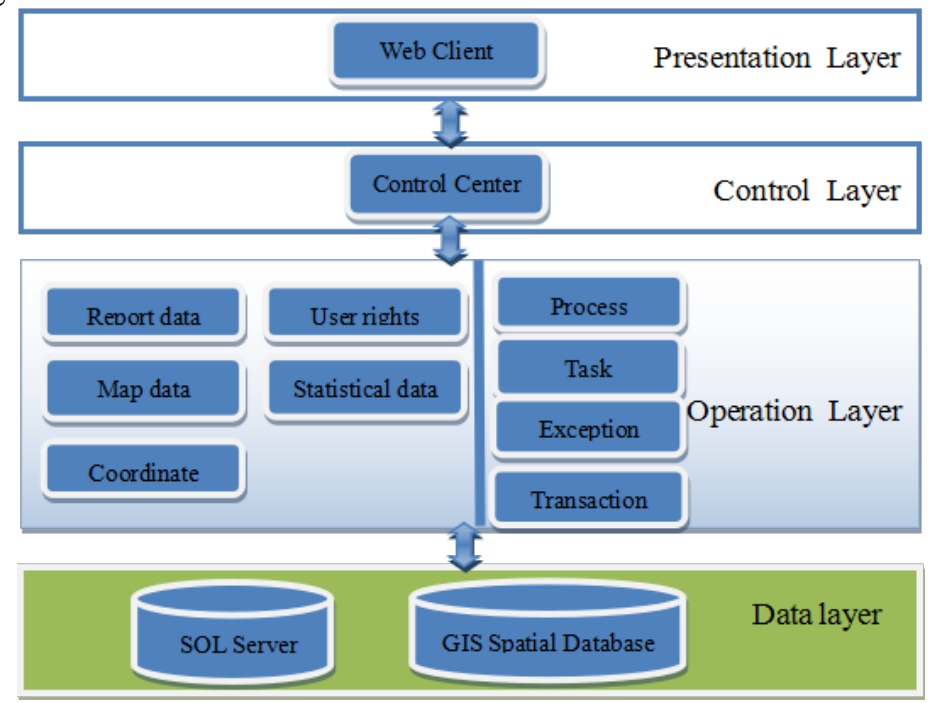

Fig. 1 The Overall Software Framework of the System

According to the structure, mobile geographic information service system can be divided into three levels, i.e., presentation layer, business logic layer and data layer. Among them, the data layer provides data support for mobile geographic information service system. It is also the source of presentation information required by this system. The business logic layer is divided into operation layer and control layer. The operation layer further encapsulates and abstracts different types of data and finally provides them for two modules, i.e. maps and graphs. The two modules correspond to two major functions of the system. They are relatively independent and favorable for the independent development of system. The control layer is the control center of data exchange. It undertakes data exchange with the presentation layer. The last is presentation layer. It finally visualizes data and presents them to users.

In correspondence, the presentation layer uses HTML5 page display technology and J2EE's corporate background to realize control and uses MSSQL database to realize data management.

HTML5 Canvas can provide a magnificent page display effect. Its powerful API library can support JS and other scripting languages perfectly and satisfy users' demands for different presentation styles of data. Moreover, the powerful corporate framework of J2EE can process the business logic required by system processing perfectly and support cross-platform operation. It has good compatibility. Finally, MSSQL database not only has good compatibility, expansibility and 
reliability, but also has the function of developing new Internet systems quickly. It can store XML data directly and output search results in XML format and so on. It is helpful to the establishment of the interoperability of heterogeneous systems. The final designed system supports the access of different terminal equipment, including mobile phones, tablets, laptops and PC and satisfies the data visualization needs of users in different scenarios. It implements mobile information services while improving the degree of user experience.

\section{Implementation of the system}

The service group of this system is mainly scientific researchers and those who are interested in scientific statistical data. These people often use scientific statistical data in their work and life. To improve the availability of data and the diversity of presentation is their urgent demand. Based on an in-depth survey of users' demands, this paper sorts out and analyzes scientific statistical data and suggests that this mobile geographic information service system should implement the following functions:

1) It combines with the data visualization service of Cartogram. Cartogram is a national geographic information public service platform and ensures the reliability and effectiveness of geographic information [4]. The system realizes the display, zoom in and zoom out, layer management and other basic map functions of Beijing maps, by using Cartogram. Combined with relevant scientific statistical data, it realized the display of dotted information on the map and hierarchical maps.

2) It realizes the independent visualization module of statistical information. It includes two kinds of forms, i.e., information reports and statistical charts. They are two different presentation methods of data. Information reports can choose any kind of information content, select corresponding field information in it and generate statistical reports, so as to display the specific content of various statistical data clearly. Statistical charts mainly include: pie charts, line charts and bar chart, etc. Among them, pie charts are used to express the proportion of every indicator. Line charts express the changes of various scientific statistical data. Bar charts express differences between various R\&D institutions or years.

Presentation of dotted information. According to their own demands, users input key words or select search fields in the search box, the system will query relevant units, according to the search criteria and display the distribution of relevant units on the map. If users want to know detailed information of a certain unit, they can click on the icon of this unit. Then the system will pop up a table to show details of this unit, as shown in Flow Chart 2:

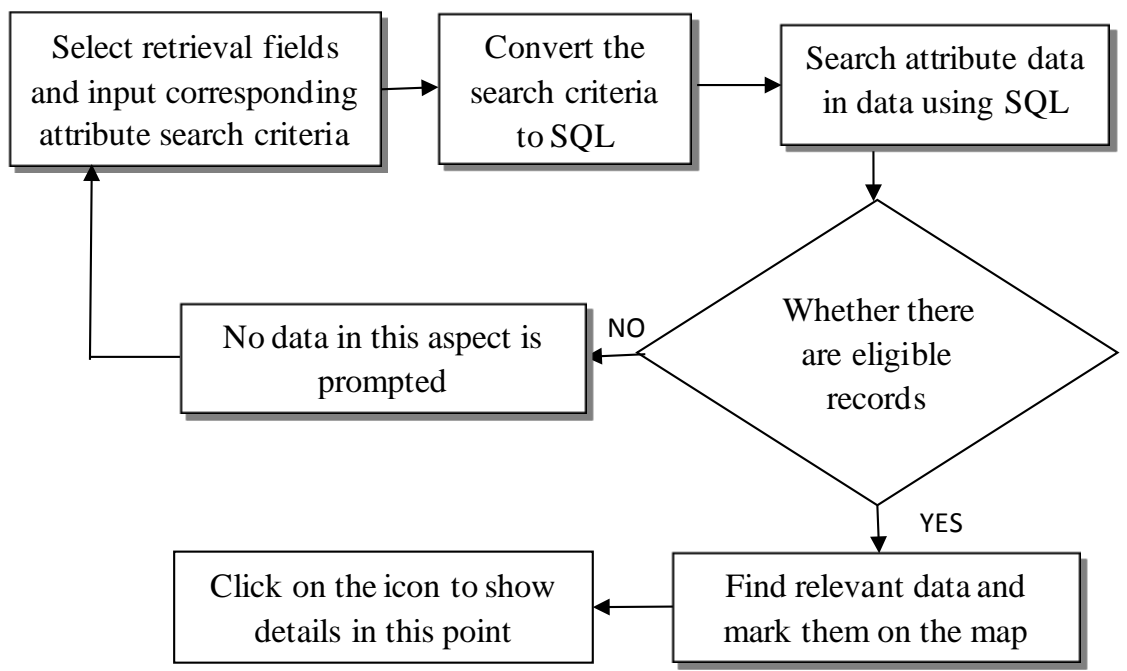

Fig. 2 The Flow Chart of Dotted Information Query

Dotted information contains the name, address, funding, scientific personnel and other information of R\&D institutions, schools and key laboratories. They system set up dotted symbols on the map by building a new layer. When clicking the symbols with mouse or finger, a table will pop up 
to display details of relevant units. This paper first defines the createIcon function and creates dotted symbols and defines the shape, size, location and other parameters of symbols in this function. Then it defines the mapLayersWithInfo function, builds a new layer, displays the dotted information and displays an event function to monitor the mouse click. When the mouse clicks on the icon, detailed information will be displayed. When clicking again, the above information can be hidden.

In the system, R\&D institutions in the first industry in Beijing are searched. The search results are displayed on the map. Click the icon on the map to show detailed information of relevant R\&D institutions, as shown in Illustration 3:

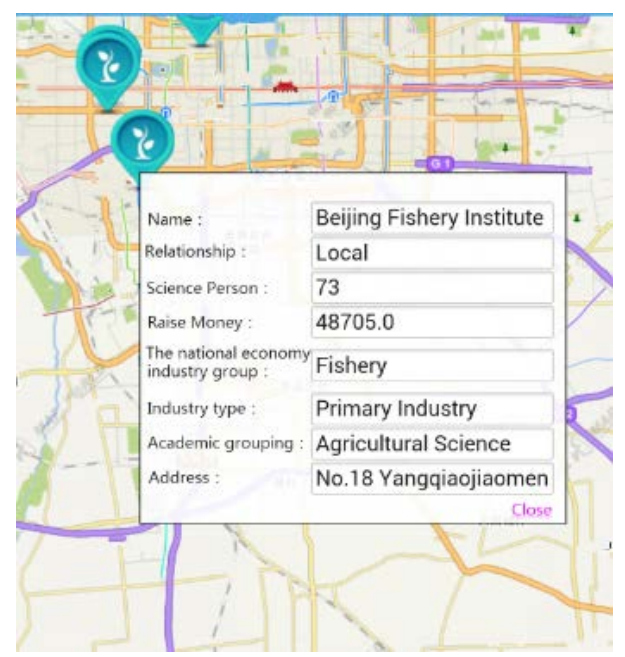

Fig. 3 The Implementation Effect of Dotted Information

Implementation of hierarchical maps. Hierarchical maps display project results, R\&D institutions, patents, technical market and other statistical data of 18 districts and counties in Beijing on the map. They use area coloring. According to the data size of various districts and counties, the higher data, the deeper color of area. In this way, a good visual effect can be achieved.

According to various indicators, the data of various districts and counties are stored in the database. When users select corresponding indicators to query data, the page uses AJAX asynchronous communication to obtain data from background and then split and save them. This paper uses the variable arrcode to save the administrative code of districts and counties, arrvalue to save corresponding data value. The system uses API in Cartogram to define the color of hierarchical maps, name parameter of display areas, line parameter, the scope of hierarchical data and other parameters. The implementation steps are as follows:

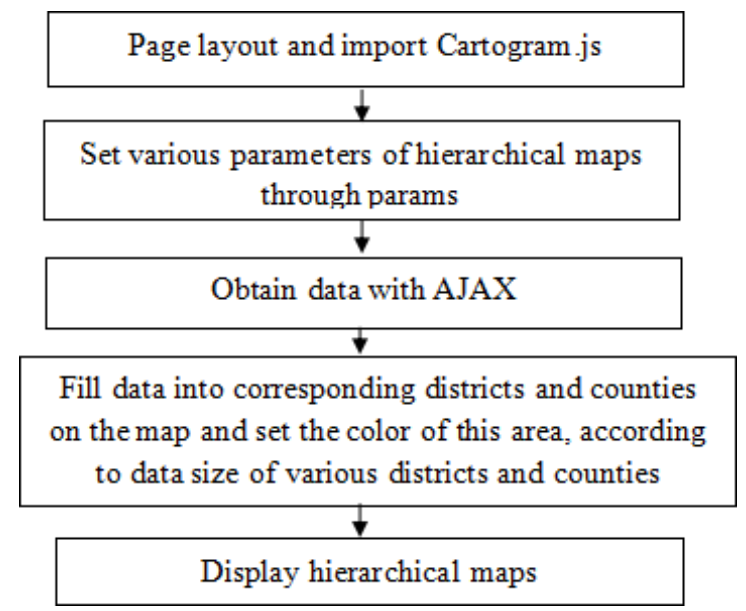

Fig. 4 The flow chart of hierarchical map implementation

The project results data of 18 districts and counties in Beijing are shown on the map. The more project results, the deeper color in these districts and counties. Otherwise, the colors are light. The illustration is shown in Figure 5: 


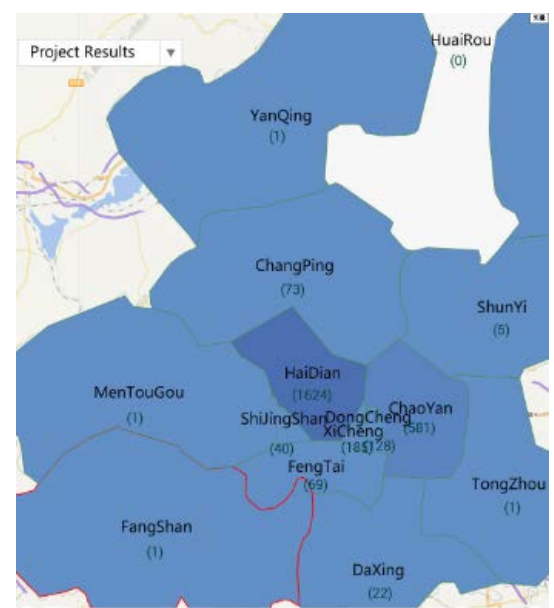

Fig. 5 The Implementation Effect of Hierarchical Maps

The visualization of statistical information. This module separates business data from GIS spatial information service platform and provides multiple kinds of business information query services, including patents, construction planning and project results, etc. This module can display data more specifically and combine with the two display methods above, allowing users to observe data more comprehensively.

The data are processed with ECharts on the front page, to achieve visual and vivid display effect. ECharts is based on HTML 5 Canvas. It is a pure JavaScript chart library and provides visual, vivid, interactive and personalized data visualization charts [5]. The mapping of statistical charts using Echarts library involves four steps: Step 1 is to introduce software developer's kit (SDK) and required topic files (you can define your own subject files), define and design the page layout, define a chart container. Step 2 is ordinary initialization charts. Charts are initialized by invoking SDKs. Step 3 is to set the option attribute to set data of charts. Option is an array element. Tooltip is a prompt box. Legend is conventional signs. Type is the type of charts (bar charts, pie charts and line charts, etc.). Calculable sets whether it can be drag-and-drop or not. Series set data, etc. Finally, Ajax is used to obtain data. After analysis, data is filled into series. The implementation effect is shown in Figure 6:

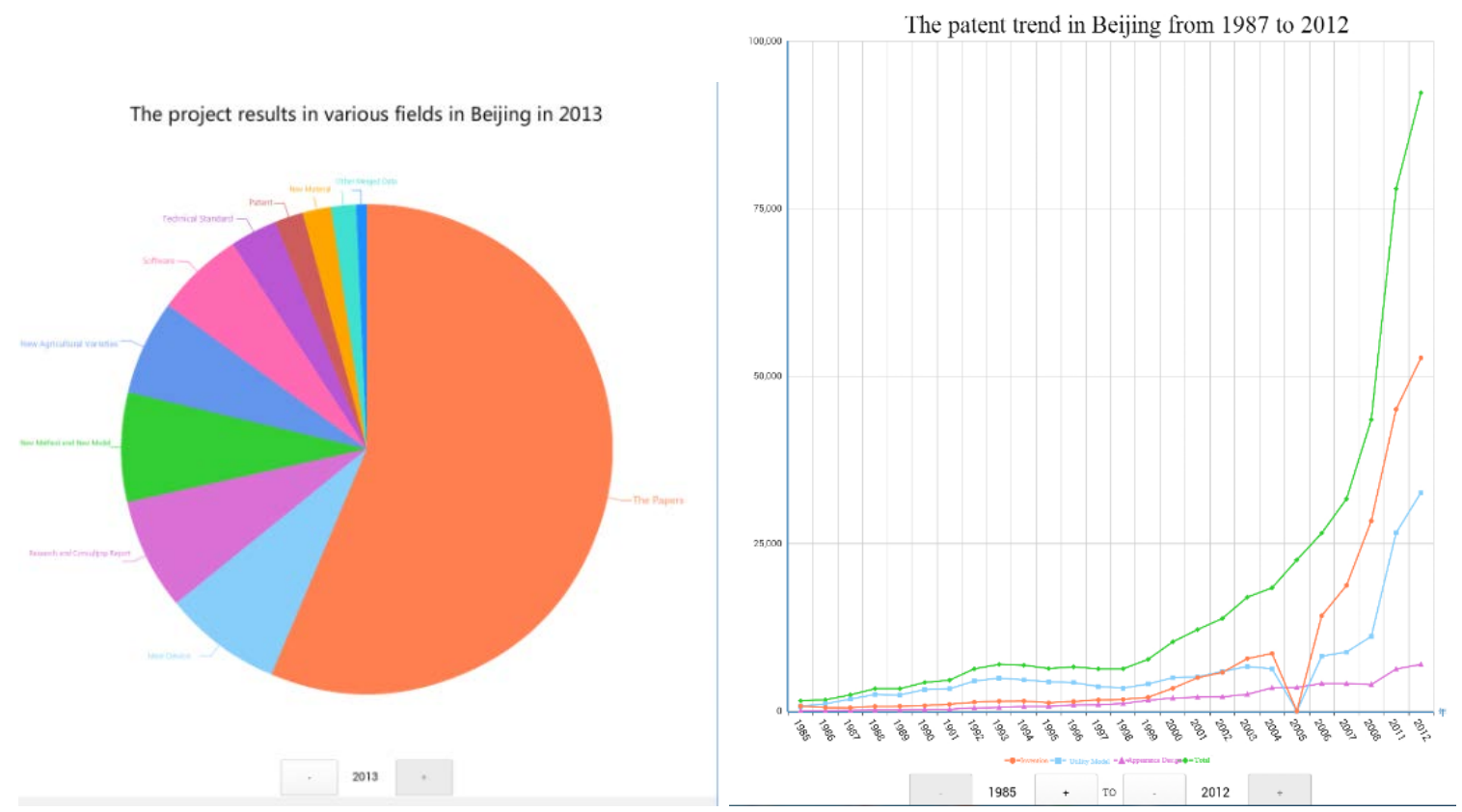

Fig. 6 Illustration of Data Visualization

The line chart is a visual presentation of patent trend in Beijing from 1987 to 2012. The pie chart is a visual presentation of project results in various fields in Beijing in 2013. 


\section{Conclusion}

This topic analyzes problems existing in the utilization of scientific statistical data. Based on the research of key technology, it designs a mobile geographic information service system based on JSP. Users can observe the distribution of $R \& D$ institutions through this system, click points of interest on the map to obtain their detailed information, or obtain visual data comparison effect through the color depth in hierarchical maps. In addition, the system designs a statistical information visualization module alone, with which users can observe more detailed and visual data. This topic solves scattered data, lack of availability, slow processing speed and other problems. It has a high application value among those who are interested in scientific statistical data. Through tests, the system has stable, and reliable operations and basically completes the requirements of project. The implementation effect is good.

\section{References}

[1] Luo, L.J., Deng, S.H. and Jin, X.F. From the Geographic Information Services to Geodesign Services [J], Geomatics World, 2012(06):40-46. In Chinese.

[2] Wei, Q. JSP technology introduction and development prospects [J], Equipment Manufacturing Technology, 2008(07):118-119+124. In Chinese.

[3] Xu, W. and Gao, J.H. Research of Web Application Framework Based on Spring MVC and MyBatis [J], Microcomputer Applications, 2012(07):1-4+10. In Chinese.

[4] Li, Z.G. Building Map World Big Data Center, Creating a National Strategic Information Basic Platform[J], Journal of Geomatics, 2015(02):10-13. In Chinese.

[5] Yang, J.Y. and Geng, J.P. A real-time Web data monitoring system based on HTML5 [J], Journal of Guilin University of Electronic Technology 2015(02):136-141. In Chinese. 